\title{
The effect of maternal sleep deprivation on differentiation of mesenchymal stem cells in the presence of neonates brain cerebrospinal fluid of Wistar rats
}

\author{
Shariat $\mathrm{SH}^{1}$, Parivar $\mathrm{K}^{1}$, Roudbari $\mathrm{NH}^{1}$, Nabiuni $\mathrm{M}^{2}$ \\ Department of Biology, Science and Research Branch, Islamic Azad University, Tehran, Iran. \\ kazem.parivar@yahoo.com
}

\begin{abstract}
OBJECTIVE: Cerebrospinal fluid (CSF) contains proliferation, differentiation and maturation signals that are essential factors for brain development. Due to presence of such factors this fluid has proliferative and differentiation potential. A previous study showed that maternal sleep deprivation (MSD) decrease the number of newborn neurons in development of hippocampus. Also, it impairs hippocampus-dependent spatial learning and memory in the young offspring rat. MSD can change CSF factors. In the present study, the effect of MSD-CSF on differentiation of mesenchymal stem cells to neural cells was examined, and expression of Nestin, Neun, and NeurD1 that are neurogenic markers was investigated.

MATERIAL AND METHODS: In this study, bone marrow mesenchymal stem cells were aspirated from the femur and tibia of young male Wistar rats. Then cell suspension was cultured in DMEM medium supplemented with $10 \%$ FBS and $1 \%$ antibiotics. Pregnant rats were subjected to sleep deprivation for 6 $\mathrm{h}$ by gentle handling during 4th, 7th, and 18th day of pregnancy. CSF was collected from cisterna magna of neonate rats. CSF was added to culture media with a $5 \%$ ratio $(\mathrm{v} / \mathrm{v})$. Then cell viability was determined with MTT assay. Total cellular RNA was extracted, cDNA was synthesized and NeuN, Nestin, NeuroD1 and IL-6 genes were analyzed by Real-time PCR.

RESULTS: Real time-PCR analysis showed that expression of Neun and NeurD1gene decreased compared with culture in normal CSF (N-CSF), and also showed that expression of Nestin did not decrease, inflammatory gene (IL-6) showed high expression compared to culture with N-CSF.

CONCLUSION: Based on our results, MSD-CSF could inhibit neurogenesis process in mesenchymal stem cells and also, this result suggests that MSD can suppress neural differentiation and decrease of neurogenesis gene expression. Overall these findings suggest that insomnia and sleep loss may active inflammatory responses in the brain and change CSF-markers (Fig. 3, Ref. 34). Text in PDF www.elis.sk. KEY WORDS: sleep deprivation, maternal sleep deprivation, CSF, stem cells, neural gene expression.
\end{abstract}

\section{Introduction}

Cerebrospinal fluid (CSF) contains a variety of pro-growth factors that can induce proliferation and differentiation of mesenchymal stem cells (MSCs) into neural cells, this suggests that CSF is a suitable inducer for neural stem cell (NSC) and can nourish the nerve cell. This research can be a reference for wide clinical application of NSCs in the future (1). Our group had similar observation in our previous studies, and the same effect was also showed that CSF contains several factors such as transforming growth factor- $\beta$ (TGF- 3 ), nerve growth factor (NGF), brain derived neurotrophic factor (BDNF), neurotrophin-3 (NT-3), insulin-like growth factor

${ }^{1}$ Department of Biology, Science and Research Branch, Islamic Azad University, Tehran, Iran, and ${ }^{2}$ Department of Cell and Molecular Biology, Kharazmi University, Tehran, Iran

Address for correspondence: K. Parivar, Dr, Department of Biology, Science and Research Branch, Islamic Azad University, Tehran, Iran, Postal Code: 1477893855 ,

Phone: +98.2144865179 , Fax: +98.2144865154
(IGF), hepatocyte growth factor (HGF), and cytokines (2), RA (3), which regulate the survival, proliferation, and differentiation of the neuroepithelium (2). Other study showed that CSF can induce differentiation of umbilical cord blood mesenchymal cells into neural cells (4). There is some discussion about the timing of formation and the effectiveness of different barrier systems within the brain. It is accepted that neural stem cells are in direct contact with CSF during most of the development stages (3). Although CSF factors encourage the proliferation and differentiation of MSCs into nerve cells, on the other hand, several studies suggested that CSF contains inhibitory element, which can suppress the differentiation of stem cells into nerve cell $(1,5)$. This fluid component is not fixed, can be altered by pathological state of central nervous system and promote these inhibitory components (6). One of the pathological states is sleep deprivation. Brain gene expression is generally changed during sleep and wakefulness in both vertebrate and invertebrate and also in rat long-term sleep deprivation causes cellular stress for neurons and glial cells and triggers a dramatic physiological change that leads to death after 2-3 weeks (7). Based on the above information, sleep deprivation can change CSF biomarkers in Al- 
zheimer disease and shows that CSF orixitin increase underexposed SD induced (8). Sleep deprivation changes physiological and psychological behavior. Also, induced changes are associated with metabolic syndrome (diabetes), hormonal changes affecting hypothalamic-pituitary-adrenal axis $(9,10)$ and increased levels of pro-inflammatory cytokines $(9,11)$. Sleep loss can have effects on health and have been shown to be a risk factor for several diseases $(12,13)$. Sleep deprivation leads to severe physical changes $(14)$ such as debt in attention, cognition, immune function, metabolism, mood, and cardiovascular function (12). Sleep deprivation effects are not limited to psychological change, they also influence human brain. Fifel and coworker have shown the impact of sleep deprivation on neuronal activity in four hypothalamic areas (15). Other investigations have shown that sleep deprivation impairs synaptic tagging in mouse hippocampal slices (16), and decreases neuronal excitability and responsiveness in rats (17). It is proved that sleep deprivation effects neuro-endocrine hormones in women during puberty, pregnancy and menopause cycle (18). Previous studies have shown that $84 \%$ of pregnant women have sleep disturbances and sleep deprivation in pregnant woman is a serious threat for newborn health, so that body weight of newborn effected by maternal sleep deprivation is low compared to control group (18). Also, anxiety disorders in offspring rise by MSD induction (19). Chang and coworkers reviewed the effect of MSD on maternal and fetal health and they reported that MSD can elevate the spontaneous preterm delivery (18). Recent assays exhibited that maternal sleep deprivation (MSD) inhibits adult hippocampus neurogenesis and impairs hippocampal dependent spatial learning and memory in young offspring rats (14). Although previous studies demonstrated that maternal sleep deprivation induced an alteration in offspring behavior (19), sexual behavior in F1 offspring (20) and impair in hippocampal long-term potentiation (LTP) that leads to memory deficit in young offspring rats (14). The effects of sleep deprivation at different stages of pregnancy and experimental offspring CSF on neurogenesis have not been extensively studied. The current study was designed to examine the effects of maternal sleep deprivation (MSD) by gentle handling in rat on differentiation of mesenchymal stem cells (MSCs) in presence of neonates brain CSF to neural lineage.

\section{Materials and methods}

All experiments were performed following an ethical review by the animal use committee of the University of Kharazmi. Wistar rats were bred in animal house of the Department of Biology, Kharazmi University. They were kept in rat boxes at constant temperature of $25^{\circ} \mathrm{C}$ and 12 hours light/dark cycle with free access to food and water. Individual male and female rats were mated in cages and checked regularly for observation of a vaginal plug to determine embryonic day 0 (E0).

\section{Sleep deprivation induction}

Sleep deprivation was induced using a modified small-platform method. Pregnant Wistar rats for sleep deprivation were individually housed in the experiment device by two platforms, platforms were $2 \mathrm{~cm}$ above the water level. The rats fell into the water if they lost muscle tone, forcing them to claim back on the platform and remain awake. All pregnant rats were in platform for 8 hours three times during pregnancy (4th-7th-18th day) and deprived of REM and NON-REM sleep. All rats had free access to food and water. After sleep deprivation the pregnant females were put back to their room individually (14).

\section{Collection of CSF}

Samples of CSF were collected from the cisterna magna of newborn rat (1 day) using glass micropipettes and capillary action without aspiration. Aspiration invariably resulted in bleeding and contamination of the samples. Offsprings were positioned with heads flexed down onto the chest to allow penetration into the cisternal cavity through the skin and underlying muscle. Samples containing undesirable blood contamination were discarded. All samples were collected into sterile microtubes and centrifuged at 14,000 RPM to remove cells or debris from the fluid, and the supernatant was transferred into another sterile tube. These samples were stored at $-80^{\circ} \mathrm{C}$ until use. The volume of CSF collected from each offspring was between 5 and $50 \mu$ and samples were pooled for each experiment (2).

\section{Bone marrow stem cells culture and in vitro tests}

Bone marrow stem cells were aspirated from the femur and tibia of young male rats (100-120 g animals) by flushing method. Then cell suspension cultured in DMEM was supplemented with FBS and antibiotics. The cultures were kept in a humidified incubator with $5 \% \mathrm{CO}_{2}$ and maintained at $37^{\circ} \mathrm{C}$. Stem cells cultured medium was refreshed in a $2 / 3$ ratio every 2 days. The cultures were divided into three groups for study. Control: No CSF exposure, N-CSF: exposure to normal offspring CSF, MSD-CSF: exposure to offspring CSF by mother under sleep deprivation. For tests, $100 \mathrm{Ml}$ of cells at $4 \times 10^{4} \mathrm{cells} / \mathrm{ml}(4000)$ cell $/$ well) in culture medium were plated into poly-D- lysine coated 96 -well plates and cultured in a DMEM without serum for 24 hours and then supplemented with CSF ( $5 \% \mathrm{v} / \mathrm{v})$ for 7 days. After one week, cells were photographed and then prepared for morphological examination and Real time-PCR.

\section{Cell viability assay}

Cell viability and/or proliferation was quantitatively determined by the MTT assay. Exposing cells to any new factors may affect cell survival. The cytotoxicity of added factors depends on the dosage. A different study has shown that even a high dose of an inducer might have a cytotoxic effect on cells $(2,21,22)$. We examined effect of MSD-CSF dosages on cell viability by MTT method using a colorimetric assay in control and medium by N-CSF and MSD-CSF. MTT (3-(4,5-dimethylthiazol-2-yl)-2,5diphenyltetrazolium bromide), is a yellow tetrazolium dye that responds to metabolic activity. Reductive enzymes in living cells reduce MTT from a pale yellow color to dark blue formazan crystals. In 24 -well plates, cells were seeded at $7 \times 10^{4}$ cell $/$ well in 500 $\mu 1$ DMEM without FBS. As described above, cells were treated with a solution of five $\mathrm{mg} / \mathrm{ml} \mathrm{MTT}$ and incubated. After $2 \mathrm{~h}$ at 37 
164-169

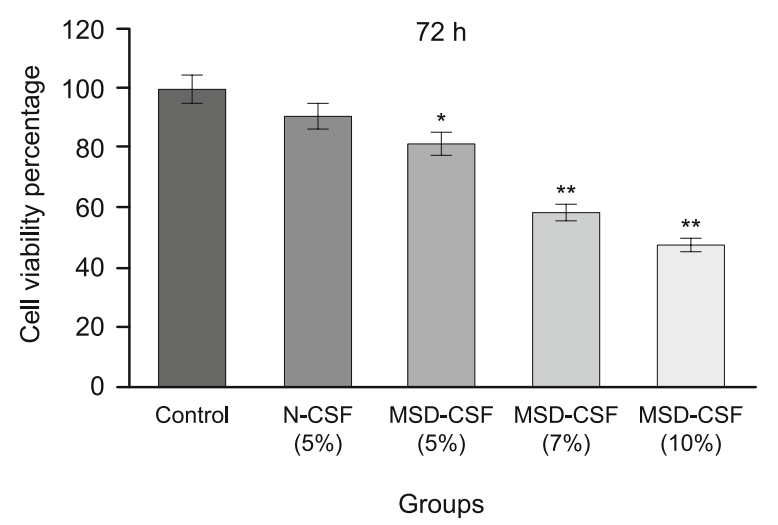

Fig. 1. Survival and/or proliferation of rat BMSCs with MSD-CSF treated by MTT assay. Data are expressed as a percentage of control (culture without CSF treat). BMSCs cultured with $5 \%$ MSD-CSF had significant viability and proliferation compared to control. Values are presented as mean $\pm \mathrm{SD}(\mathrm{n}=8) * \mathrm{p}<0.05, * * \mathrm{p}<0.01$

${ }^{\circ} \mathrm{C}$ crystals were dissolved in Triton X-100 $10 \%$. Absorbance was read at wavelength of $570 \mathrm{~nm}$.

\section{Measurement of neuritis length}

Cells in each culture were photographed with a phase-contrast microscope to visualize outgrowths from the cells and sizes of neurons were evaluated by Image $\mathrm{J}$ software.

\section{RNA extraction and RT-PCR analysis}

Total cellular RNAs exposed with normal and MSD-CSF BMSCs culture were isolated using RNA extraction kit (Qiagen, Germany (Qiagen is a provider of sample and assay technologies for molecular diagnostics, applied testing, academic and pharmaceutical research)) on the basis of protocol instruction. Total RNA was reverse-transcribed to cDNA using the cDNA synthesis kit (Qiagen, Germany). RT-PCR was performed 1 cycle reverse transcription at $95^{\circ} \mathrm{C}$ 4 min, and 35 cycles as denaturation at $94{ }^{\circ} \mathrm{C}$ for $30 \mathrm{~s}$, annealing at $59{ }^{\circ} \mathrm{C}$ for $30 \mathrm{~s}$, extension at 72 ${ }^{\circ} \mathrm{C}$ for $30 \mathrm{~s}$ and 1 cycle $5 \mathrm{~min}$ at $72{ }^{\circ} \mathrm{C}$. The values were normalized against GAPDH internal control gene. The primer sequences were as follow:

NeurD1 5'CCTACTCCTACCAGTCCCCT3' as the forward primer and 5, CAAACTCGGTGGATGGTTCG3' as reverse primer; nestin 5'GTTTTGGGTAAGAG CTGGCC3' as forward primer and 5'TCCTGTCCTAGCCCCATACT3' as reverse NeuN; 5'AGCTGAATATGCCCCACCTC3' as forward primer and GGTGGAGTTGCTGGCTATCT as reverse primer. IL-6 5'GTCAGGGGTGGTTATTGCAT3' as forward primer and 5'AGTGAGGAACAAGCCAGAGC3' as reverse. And oligonucleotide 5'-ATCACTGCCACCCAGAAGA-3' as GAPDH forward and 5'-ATGAGGTCCACCACCCTGTT-3' reverse were used for amplification of GAPDH as an internal animal control, giving a product size of $443 \mathrm{bp}$. Fold change in RNA levels was calculated using the $\Delta \Delta \mathrm{Ct}$ method (relative quantitation), and $18 \mathrm{~S}$ rRNA expression was used as an internal control.

\section{Statistical analysis}

All values were expressed as mean \pm error of the mean (SEM). Statistical analysis was performed using the one-way ANOVA and Tukey test. A level of $\mathrm{p}<0.05$ was considered statistically significant

\section{Results}

Effects of maternal sleep deprivation CSF (MSD-CSF) on survival and proliferation of BMSCs

The dose-effect of MSD-CSF on rat BMSC was assessed by MTT analyzes. MTT assay was used to quantify cell proliferation and viability. The cells were cultured by MSD-CSF for $72 \mathrm{~h}$ by 5-7 $10 \% \mathrm{~V} / \mathrm{v}$ dosage. As shown in Figure 1 cell by $10 \%$ treatment indicated remarkable cytotoxicity under MSD-CSF.

\section{Effect of MSD-CSF on the proliferation and differentiation of rat BMSCS}

We evaluated the influence of sleep deprivation at different stages of pregnancy days $4,7,18$ on proliferation and differentiation of BMSCs treated by offspring CSF. We first cultured BM$\mathrm{SCs}$ in medium supplemented. We aimed to demonstrate that offspring CSF has a direct role in neurogenesis, so we add normal CSF to BMSc for $72 \mathrm{~h}$ (Fig. 2A) when these cells were cultured by medium supplemented with normal CSF, we observed neural
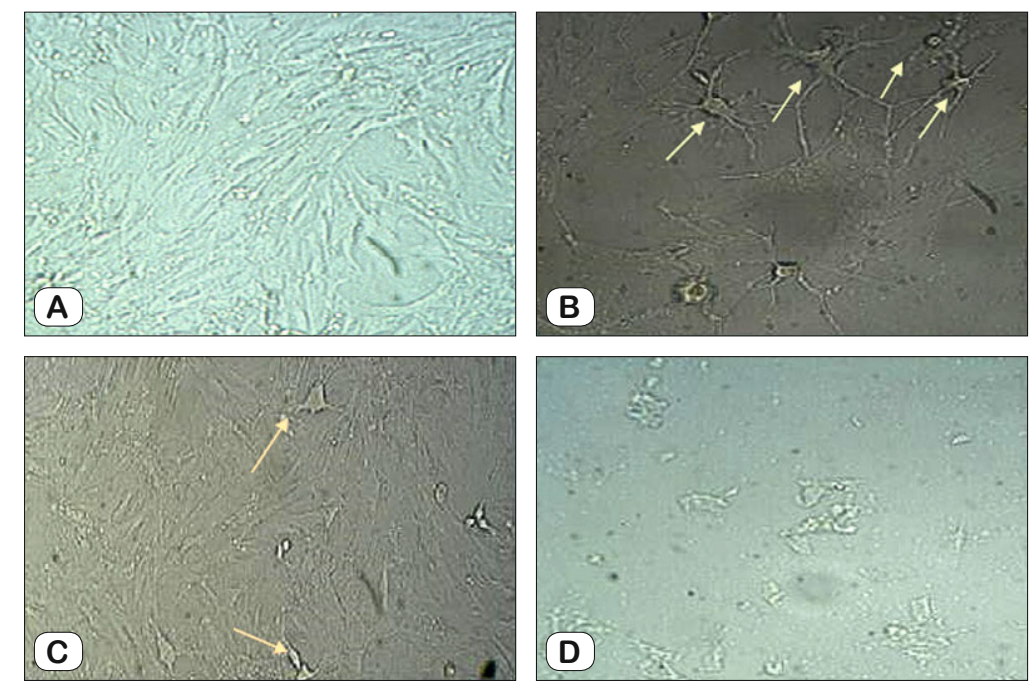

Fig. 2. The effect of N-CSF and MSD-CSF on BMSCs cells. A: BMSCs untreated cells, B: N-CSF (5 \% v/v) BMSCs treated cells, C: MSD-CSF (5\% v/v) BMSCs treated cells and MSD-CSF (10 \% v/v) BMSCs treated cells. As shown, N-CSF induced neurite outgrowth and neural differentiation meanwhile MSD-CSF (5 \% v/v) didn't exhibit significant anti-proliferative and differentiation effects. In addition, MSD-CSF (10 \% $\mathrm{v} / \mathrm{v}$ ) induced significant toxicity on BMSCs cells. $\times 400$ 

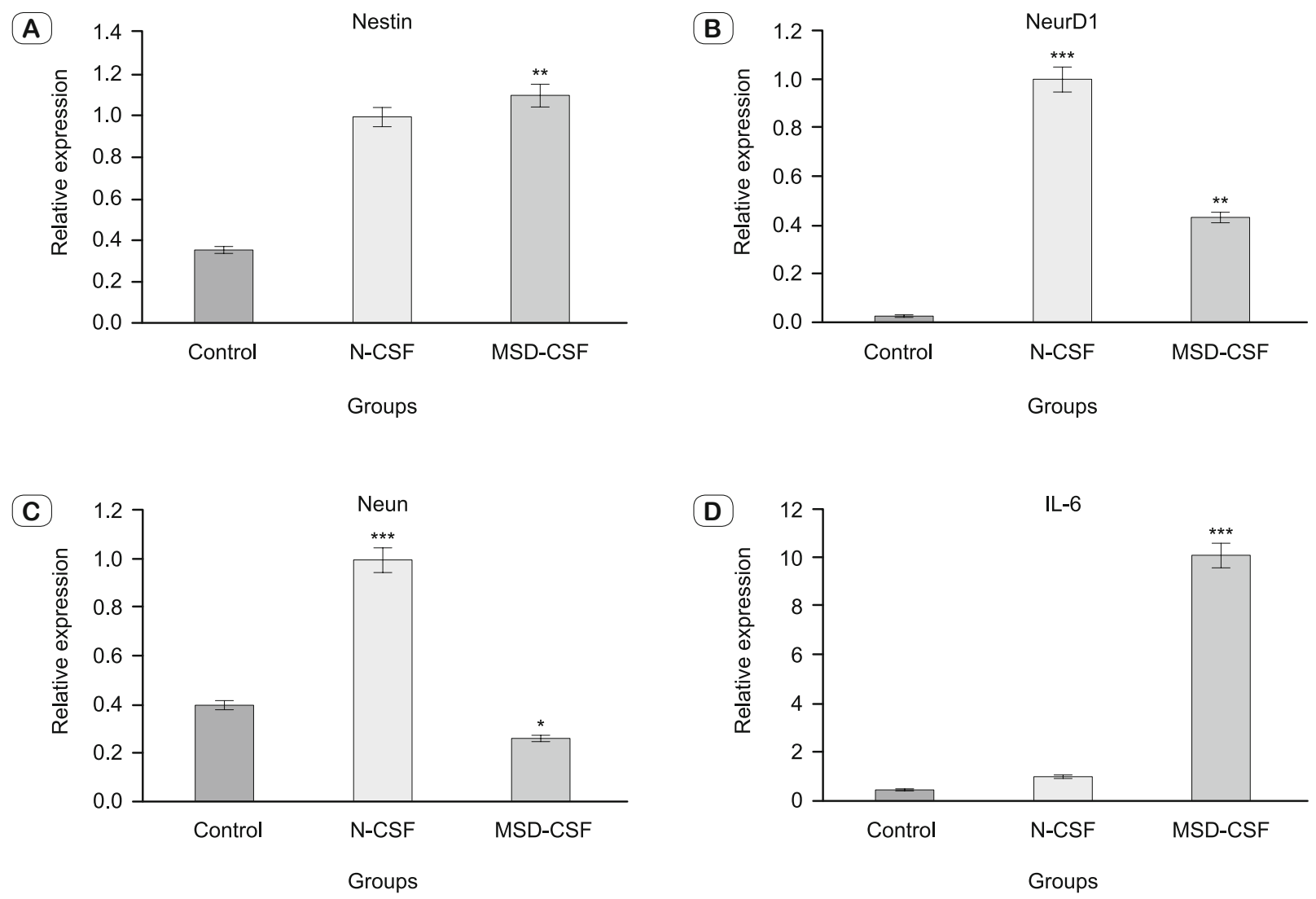

Fig. 3. An effect of BMSD-CSF on Nestin. Nestin gene enhancer activity in neural stem/progenitor cells. BMSD-CSF cannot decrease expression of Nestin gene. Data are mean $* * * \mathbf{p}<\mathbf{0 . 0 0 1}$. B and C. Effect of MSD-CSF on expression of Neun and NeurD1. Significant differences between cultures by MSD-CSF treat were detected compared with control group. Data are mean $* * *, p<0.0001$. D. Inflammatory gene. Expression of IL-6 in culture with MSD-CSF. Data are mean $* * *, p<0.001$ compared to control culture. The data are represented as the mean \pm SEM ( $n=8$ ).

cell line differentiation (Fig. 2B). In contrast, when rat BMSCs treated by MSD-CSF extract from offspring Cisterna Magna, BMSC differentiation and maturation were decreased surprisingly (Fig. 2C).

\section{qRT-PCR analyze}

As Nestin, NeuN, NeurD1 are relatively genes on CNS proliferation, differentiation and maturation they present in neural cells we employed a qRT-PCR test to detect expression of them in rat BMSCs culture. In this study, Real-time PCR assay was used to demonstrate the differentiation of mesenchymal cells to neural cells. The result revealed that MSD-CSF can induce the proliferation of BMCSc and expression of Nestin was up-regulated (Fig. 3A). However, NeuN and NeurD1 were down-regulated in MSD group. We examined whether the addition of MSD-CSF, isolated from offspring could modify proliferation and differentiation in BMSc. Based on pervious study and differentiation of MSCs to neuroprogenitor cells through exposure to CSF, we found that addition of MSD-CSF significantly decreased neural differentiation (Fig. 3) $* * * p<0 / 001$. MSD-CSF strongly reduced NeuN and NeurD1 expression compared with control group.
MSD-CSF increased expression of the inflammatory gene in MSCs culture

IL-6 is one of the inflammatory cytokines. Figure 3D shows an increase of IL-6 gene expression during underexposure with MSD-CSF compared to control. To determine the changes in proinflammatory cytokine gene expression that might be a response to sleep deprivation, these results suggest that MSD-CSF may play an important role in up-regulating inflammatory cytokines. We performed gene expression profiling using RT-PCR assays. Data analysis showed significantly up-regulated IL-6 ( $p<0.0001 * * *)$.

\section{Discussion}

Examination of sleep deprivation during human pregnancy is almost impossible (14). Therefore, the present study was designed to consider the effect of MSD-CSF on neurogenesis. In our previous study, we elucidated the direct influence of Embryonic-CSF and fetal-CSF on neurogenesis activity $(23,24)$. This research work is the first report of influence of MSD-CSF in BMSCs culture (in Vitro). According to our previous study we demonstrated cisternal CSF contains neural proliferation, differentiation and 


\section{4-169}

maturation signals (2). This study is based on qRT-PCR assay, we showed that MSD attenuated the expression of differentiation and maturation of genes (NeurD1, Neun) as compared with control groups (Fig. 3B, C). NeuN is a post-mitotic neuron marker that is highly conserved in different species (25). NeuN is a useful marker of mature neurons and causes neurogenesis in stem cells (26). This marker is required for hippocampal circuit balance and function (27). NeurD1 is essential for survival and maturation of adult-born neurons (28). NeurD1 is expressed in late stage of neurogenesis and plays an important role in differentiation, maturation and survival of neurons (28). Nestin is a neural stem/ progenitor cell marker that appears during development of the central nervous system (CNS), and it was defined as a class VI intermediate filament protein (29). Therefore, we can not assign a significant decrease in proliferative stage (Nestin expression) (Fig. $3 \mathrm{~A})$. Nestin is generally recognized as a marker protein of undifferentiated CNS cells $(29,30)$. Other study showed that the neural stem/progenitor cell marker Nestin is expressed in proliferative endothelial cells, but not in mature vasculature (29). It would be of great value to test MSD-CSF and consider its effect on differentiation of rat BMSCs to neuronal, this suggests that maternal sleep deprivation may only decrease differentiation and maturation of BMSCs to neurons but not proliferation of MSCs. This can be due to changes in CSF biomarkers, which is not consistent with a previous report that 5-8 consecutive nights of partial sleep deprivation (PSD) had no effect on CSF biomarkers for neuronal injury and astroglial activation (8). Given this controversy, further studies are required to identify a modified CSF markers inducing total sleep deprivation. There is also evidence that revealed significant effects of sleep deprivation are changes in gene expression, which can alter neuronal function. Sleep deprivation influences the immediate early genes and up-regulates expression of them. Costa and Ribeiro (31) reported that gene expression and protein level of neurotrophic factors are affected by sleep deprivation. In addition, sleep deprivation can change expression of genes related to protein synthesis and level of serum protein changes after sleep deprivation (31). Thus alteration of gene expression induced by MSD in the present study may contribute to decreased differentiation of BMSc to neural cells. Previous studies demonstrated that MSD inhibits hippocampal neurogenesis associated with inflammatory response in young offspring rats (32). It has been documented that prenatal stress impairs hippocampus dependent spatial memory (33). Further, recently it has been shown that maternal stress leads to memory deficits and disability of hippocampal synaptic plasticity in the offspring (32). Thus the effect of critical stress resulting from total sleep deprivation (TSD) can be caused by IL-6 up-regulation. In consistence with this result, we indicated that MSD increases expression of IL-6 as inflammatory marker (Fig. 3D). Interleukin 6 (IL-6) is one of cytokine family that contributes to inflammatory response. Secretion of inflammatory cytokines plays a central role as modulators of NSC niches in different processes (34). SD increases levels of inflammatory markers such as IL-6/ IL-2/ IL-1/ TNF $\alpha$ and c-creative protein (34). It elucidated that pro-inflammatory cytokines IL-1B, IL-6, $\mathrm{TNF} \alpha$ were increased after MSD induction (32). In addition, more recent researches revealed that long-term sleep deprivation triggers a stress and inflammatory response in the brain and changes in brain stress-related gene expression (31). Cireli and coworkers have shown that after short-term and long-term sleep deprivation, a number of genes are down-regulated, for instance myelin protein (7), on the other hand stress leads to gene up-regulation (31). Notably, although we used gentle handling that minimizes stress to perform maternal sleep deprivation three times during pregnancy (4th-7th-18th day of pregnancy), it may be a stressful procedure. In full agreement with this finding, we report that maternal sleep deprivation at the different stages of pregnancy displays a down-regulation of NeurD1, NeuN and up-regulation of Nestin and IL-6 gene expressions.

\section{Conclusion}

In the present study, we investigated for the first time the effect of MSD on newborn CSF and effect of MSD-CSF on BMSCs neural differentiation. We showed that MSD can suppress neural differentiation and decrease neurogenesis genes. Overall these findings suggest that insomnia and sleep loss may activate inflammatory responses in the brain and change CSF-marker. Future studies will be required to determine the most important CSF-marker that is changed by MSD and detailed mechanism of action of MSD-CSF on neurogenesis.

\section{References}

1. Ren C, Yin P, Ren N, Wang Z, Wang J, Zhang C et al. Cerebrospinal fluid-stem cell interactions may pave the path for cell-based therapy in neurological diseases. Stem Cell Res Ther 2018; 9 (1): 66.

2. Nabiyouni M, Storey C, Owen-Lynch J, Miyan J. Proteomics study of CSF composition in the developing H-Tx rat. Cerebrospinal Fluid Res 2004; 1 (S1): S29.

3. Alonso MI, Martín C, Carnicero E, Bueno D, Gato A. Cerebrospinal fluid control of neurogenesis induced by retinoic acid during early brain development. Dev Dyn 2011; 240 (7): 1650-1659.

4. Farivar S, Mohamadzade Z, Shiari R, Fahimzad A. Neural differentiation of human umbilical cord mesenchymal stem cells by cerebrospinal fluid. Iran J Child Neurol 2015; 9 (1): 87.

5. Babavalian H, Latifi AM, Shokrgozar MA, Bonakdar S, Tebyanian H, Shakeri F. Cloning and expression of recombinant human plateletderived growth factor-BB in Pichia Pink. Cell Mol Biol (Noisy-le-grand) 2016; 62 (8): 45-51.

6. Hashemi E, Sadeghi Y, Aliaghaei A, Seddighi A, Piryaei A, Broujeni ME et al. Neural differentiation of choroid plexus epithelial cells: role of human traumatic cerebrospinal fluid. Neural Regen Res 2017; 12 (1): 84.

7. Cirelli C, Faraguna U, Tononi G. Changes in brain gene expression after long-term sleep deprivation. J Neurochem 2006; 98 (5): 1632-1645.

8. Olsson M, Ärlig J, Hedner J, Blennow K, Zetterberg H. Sleep deprivation and cerebrospinal fluid biomarkers for Alzheimer's disease. Sleep 2018; 41 (5).

9. AlDabal L, BaHammam AS. Metabolic, endocrine, and immune consequences of sleep deprivation. The open respiratory medicine journal 2011; 5: 31 . 
10. Vargas I, Lopez-Duran N. Investigating the effect of acute sleep deprivation on hypothalamic-pituitary-adrenal-axis response to a psychosocial stressor. Psychoneuroendocrinology 2017; 79: 1-8.

11. Taherian A, Fazilati M, Moghadam AT, Tebyanian H. Optimization of purification procedure for horse $\mathrm{F}\left(\mathrm{ab}^{\prime}\right) 2$ antivenom against Androctonus crassicauda (Scorpion) venom. Trop J Pharm Res 2018; 17 (3): 409-414.

12. Lim J, Dinges DF. A meta-analysis of the impact of short-term sleep deprivation on cognitive variables. Psychol Bull 2010; 136 (3): 375.

13. Ma N, Dinges DF, Basner M, Rao H. How acute total sleep loss affects the attending brain: a meta-analysis of neuroimaging studies. Sleep 2015; 38 (2): 233-240.

14. Peng Y, Wang W, Tan T, He W, Dong Z, Wang YT et al. Maternal sleep deprivation at different stages of pregnancy impairs the emotional and cognitive functions, and suppresses hippocampal long-term potentiation in the offspring rats. Mol Brain 2016; 9 (1): 17.

15. Fifel K, Meijer JH, Deboer T. Long-term effects of sleep deprivation on neuronal activity in four hypothalamic areas. Neurobiol Dis 2018; 109: 54-63.

16. Vecsey CG, Huang T, Abel T. Sleep deprivation impairs synaptic tagging in mouse hippocampal slices. Neurobiol Learn Mem 2018; 154 : 136-140.

17. Borbély S, Világi I, Haraszti Z, Szalontai Ö, Hajnik T, Tóth A et al. Sleep deprivation decreases neuronal excitability and responsiveness in rats both in vivo and ex vivo. Brain Res Bull 2018; 137:166-177.

18. Chang JJ, Pien GW, Duntley SP, Macones GA. Sleep deprivation during pregnancy and maternal and fetal outcomes: is there a relationship? Sleep Med Rev 2010; 14 (2): 107-114.

19. Calegare BFA, Fernandes L, Tufik S, D'Almeida V. Biochemical, biometrical and behavioral changes in male offspring of sleep-deprived mice. Psychoneuroendocrinology 2010; 35 (5): 775-784.

20. Alvarenga TA, Aguiar MFP, Mazaro-Costa R, Tufik S, Andersen ML. Effects of sleep deprivation during pregnancy on the reproductive capability of the offspring. Fertil Steril 2013; 100 (6): 1752-1757.

21. Seifi Kafshgari H, Yazdanian M, Ranjbar R, Tahmasebi E, Mirsaeed S, Tebyanian H, Ebrahimzadeh MA, Goli HR. The effect of Citrullus colocynthis extracts on Streptococcus mutans, Candida albicans, normal gingival fibroblast and breast cancer cells. J Biol Res 2019; 92 (1).

22. Soufdoost RS, Yazdanian M, Tahmasebi E, Yazdanian A, Tebyanian H, Karami A et al. In vitro and in vivo evaluation of novel Tadalafil $/ \beta-\mathrm{TCP} /$ Collagen scaffold for bone regeneration: A rabbit critical-size calvarial defect study. Biocybernetics and Biomedical Engineering 2019 2019/07/20/.
23. Nabiuni M, Rasouli J, Parivar K, Kochesfehani HM, Irian S, Miyan JA. In vitro effects of fetal rat cerebrospinal fluid on viability and neuronal differentiation of PC12 cells. Fluids Barriers CNS 2012; 9 (1): 8.

24. Yari S, Parivar K, Nabiuni M, Keramatipour M. Effect of embryonic cerebrospinal fluid on proliferation and differentiation of neuroprogenitor cells. Cell J 2013; 15 (1): 29.

25. Duan W, Zhang Y-P, Hou Z, Huang C, Zhu H, Zhang C-Q et al. Novel insights into NeuN: from neuronal marker to splicing regulator. Mol Neurobiol 2016; 53 (3): 1637-1647.

26. Lind D, Franken S, Kappler J, Jankowski J, Schilling K. Characterization of the neuronal marker $\mathrm{NeuN}$ as a multiply phosphorylated antigen with discrete subcellular localization. J Neurosci Res 2005; 79 (3): 295-302.

27. Wang H-Y, Hsieh P-F, Huang D-F, Chin P-S, Chou C-H, Tung C-C et al. RBFOX3/NeuN is required for hippocampal circuit balance and function. Scientific reports 2015; 5: 17383.

28. Gao Z, Ure K, Ables JL, Lagace DC, Nave K-A, Goebbels S et al. Neurod 1 is essential for the survival and maturation of adult-born neurons. Nat Neurosci 2009; 12 (9): 1090.

29. Suzuki S, Namiki J, Shibata S, Mastuzaki Y, Okano H. The neural stem/progenitor cell marker nestin is expressed in proliferative endothelial cells, but not in mature vasculature. J Histochem Cytochem 2010; 58 (8): 721-730.

30. Heidari MF, Arab SS, Noroozi-Aghideh A, Tebyanian H, Latifi AM. Evaluation of the substitutions in 212,342 and 215 amino acid positions in binding site of organophosphorus acid anhydrolase using the molecular docking and laboratory analysis. Bratisl Lek Listy 2019; 120 (2): 139-143.

31. da Costa Souza A, Ribeiro S. Sleep Deprivation and Gene Expression. In: Meerlo P, Benca RM, Abel T, editors. Sleep, Neuronal Plasticity and Brain Function. Berlin, Heidelberg: Springer Berlin Heidelberg, 2015: 65-90.

32. Zhao Q, Peng C, Wu X, Chen Y, Wang C, You Z. Maternal sleep deprivation inhibits hippocampal neurogenesis associated with inflammatory response in young offspring rats. Neurobiol Dis 2014; 68: 57-65.

33. Aziz NHKA, Kendall DA, Pardon MC. Prenatal exposure to chronic mild stress increases corticosterone levels in the amniotic fluid and induces cognitive deficits in female offspring, improved by treatment with the antidepressant drug amitriptyline. Behav Brain Res 2012; 231 (1): 29-39.

34. Irwin MR, Wang M, Campomayor CO, Collado-Hidalgo A, Cole S. Sleep deprivation and activation of morning levels of cellular and genomic markers of inflammation. Arch Intern Med 2006; 166 (16): 1756-1762.

Received July 19, 2019. Accepted October 1, 2019. 